\title{
Micromechanism of cumulative damage in bearing steels under Rolling Contact Fatigue
}

\author{
Yachao Sun $^{1}$ and Hongrui Cao ${ }^{1}$ \\ ${ }^{1}$ Xi'an Jiaotong University
}

December 23, 2020

\begin{abstract}
Rolling Contact Fatigue (RCF) is harmful and inevitable to bearings and usually results in the initiation of subsurface damage. This paper focuses on the cumulative mechanism of subsurface damage in bearing steels arose from cementite during RCF. The distribution of subsurface shear stress in bearings was investigated by finite element simulations. A two-phase atomic model of bcc-Fe and cementite was built. Ten alternating shear load cycles were applied when the model was initially in the elastic, elastic-plastic and plastic stages, respectively. The results show that cyclic softening diversely occurs in all three types of stress responses, and the progress of plastic accumulation depends on the amplitude of cyclic load and cycles. Severe shear deformation eventually leads to the damage of the cementite phase, which might be the microscopic mechanism of the fatigue failure of bearing steels. The conclusions presented have general applicability to brittle inclusions in bearing steels.
\end{abstract}

\section{Micromechanism of cumulative damage in bearing steels under Rolling Contact Fatigue}

Yachao Sun, Hongrui Cao*

State Key Laboratory for Manufacturing Systems Engineering, Xi'an Jiaotong University, Xi'an 710049, PR China

*Corresponding author.

E-mail address: chr@mail.xjtu.edu.cn (H. Cao).

Abstract:Rolling Contact Fatigue (RCF) is harmful and inevitable to bearings and usually results in the initiation of subsurface damage. This paper focuses on the cumulative mechanism of subsurface damage in bearing steels arose from cementite during RCF. The distribution of subsurface shear stress in bearings was investigated by finite element simulations. A two-phase atomic model of bcc-Fe and cementite was built. Ten alternating shear load cycles were applied when the model was initially in the elastic, elastic-plastic and plastic stages, respectively. The results show that cyclic softening diversely occurs in all three types of stress responses, and the progress of plastic accumulation depends on the amplitude of cyclic load and cycles. Severe shear deformation eventually leads to the damage of the cementite phase, which might be the microscopic mechanism of the fatigue failure of bearing steels. The conclusions presented have general applicability to brittle inclusions in bearing steels.

Keywords: Bearing steels, Cyclic deformation, Cumulative fatigue damage, Micromechanism

\section{NOMENCLATURE}

$a_{0}$ lattice parameter of bcc-Fe

$a, b, c$ lattice parameters of cementite

$d$ distance from the maximum load point 
d1 displacement load of $7.2 \AA$

$\mathrm{d} 2$ displacement load of $8.4 \AA$

d3 displacement load of $11.4 \AA$

$L$ total dislocation length

$L_{x}$ model length

$L_{y}$ model width

$L_{z}$ model height

$L_{z z}$ model height without boundarys

$p(d)$ contact load

$p_{\max }$ maximum contact load

$V$ volume of crystal

$\tau_{\max }$ maximum orthogonal shear stress

$\tau_{y \max }$ maximum shear stress along the $y$-axis

$\rho$ dislocation density

$\gamma$ shear strain

\section{Introduction}

Fatigue failure in bearings involves in the initiation and propagation of damage originating from contact surface or subsurface. ${ }^{1,2}$ Although surface damage, such as wear, can be relieved by careful surface treatment and effective lubrication, subsurface failure caused by alternating shear stress is inevitable under the theoretical framework of Palmgren and Lundberg. ${ }^{3}$ This mode of fatigue failure is Rolling Contact Fatigue (RCF). RCF refers to a localized cumulative damage that occurs between two tangent and cyclically loaded parts. ${ }^{4,5}$ The raceways and rolling elements of a normal rolling bearing are generally subjected to billions of rolling contacts during service. With the increase of service time, cumulative damage gradually develops at a depth of 200-400 $\mu \mathrm{m}$ under contact surface, ${ }^{6}$ eventually leading to the failure of bearings. The initiation of damage prefers to appear when the region corresponding to the peak of alternating shear stress coincides with the defect region in materials, and further results in fatigue failure such as macroscopic spalling. ${ }^{7,8}$

Under the assumption of macroscopic homogeneity, bearing steels will not suffer from plastic deformation and damage during nominal elastic loading. However, the presence of phase interfaces and carbide inclusions introduces heterogeneity at microstructure level. Local yielding and micro-plastic strain accumulation will occur within bearing steels under RCF. And such micro-plastic flow is often referred to as 'ratcheting' phenomenon. ${ }^{2,9,10}$ The damage evolution of RCF is generally divided into three stages. In stage I, microplastic strain accumulation tends to occur near the inclusions, which indicates the starting point of damage initiation. ${ }^{11}$ Such damage evolution behavior is inconducive to the fatigue life of bearing steels.

The typical components of bearing steels consist of tempered martensite, homogeneously distributed primary spheroidized carbide, tempered carbide and retained austenite. ${ }^{12,13}$ Among them, martensite is a supersaturated solid solution formed by the solubilization of carbon in $\alpha$-Fe matrix. ${ }^{14}$ In addition to that, sparsely proportional carbon atoms have little influence on plastic deformation. And the crystal structure of martensite is body-centered tetragonal, ${ }^{15}$ which is similar with the body-centered cubic structure of $\alpha$-Fe, so martensite phase can be replaced by $\alpha$-Fe phase to simplify the research. The carbides in bearing steels can be categorized into metallic and non-metallic, among which the main metallic elements of metallic carbides are $\mathrm{Cr}$, Fe and Mo. ${ }^{16}$ Although the content of carbide can be reduced by fine processing and heat treatment, there still exists a certain proportion of Fe-rich $\mathrm{M}_{3} \mathrm{C}$. The primary spheroidized carbide in AISI 52100 bearing 
steels is $(\mathrm{Fe}, \mathrm{Cr})_{3} \mathrm{C} \cdot{ }^{13}$ Bhadeshia ${ }^{17}$ found that $3-4 \%$ of cementite cannot be dissolved in the process of austenization during the quenching of AISI 52100 bearing steels. Lian et al. ${ }^{18}$ found a small amount of Fe-rich $\mathrm{M}_{3} \mathrm{C}$ when studying the dissolution and precipitation of carbides in carburized M50NiL bearing steels. Due to the mismatch of two phases within materials, dislocations will be generated and emitted on the interface during deformation, thus causing plastic accumulation. ${ }^{19,20}$ Therefore, the mechanism of fatigue failure can be revealed to some extent by studying the influence of the interface between bcc-Fe and cementite on cyclic plastic accumulation in bearing steels.

Existing relevant atomic models mostly focus on the generation and evolution of defects at ferrite-cementite interface under monotonic loading. Ferrite is a kind of solid solution formed by carbon atoms occupying the interstitial space among the lattice atoms of $\alpha$-Fe crystal, and the maximum solubility of carbon in $\alpha$-Fe is only $0.022 \mathrm{wt} \%$ below $1000 \mathrm{~K} .{ }^{21}$ Therefore, ferrite phase is usually replaced by $\alpha$-Fe phase in the existing models. Ghaffarian et al. ${ }^{22,23}$ have reported that the deformation mechanism of ferrite-cementite interface strongly depends on the size, temperature and different loading directions of cementite, and have revealed the hindrance mechanism of cementite layer on dislocation, as well as the influence of temperature and lamellar thickness on the ductility of the model. Guziewski et al. ${ }^{24}$ have noted that the mechanical properties of ferrite-cementite interface depend on the volume ratio and orientation relationships (ORs) between ferrite and cementite phases. More recently, Liang et al. ${ }^{20}$ have reported the nucleation and evolution mechanism of dislocation at the ferrite-cementite interface, and have explained the plastic mechanism of fatigue failure under cyclic tensile and compressive loadings in pearlite. However, few existing papers pay attention to the initiation and evolution of interfacial defects under cyclic alternating shear load. In addition to that, Pandkar et al. ${ }^{25}$ have revealed the accumulation of deformation and micro-plastic strain during RCF due to ratcheting behavior by using finite element method, and have explained the contribution of carbide particles towards ratcheting. Nevertheless, the macroscale studied is insufficient to explain the origin of cumulative damage. And due to the limitations of spatial-temporal resolution of existing experimental instruments and the complexity of atomic modeling of bearing steels, the mechanism of plastic accumulation near inclusion in bearing steels still remains unclear.

In the present work, we focus on the plastic accumulation induced by the interface between bcc-Fe and cementite under cyclic alternating shear stress and attempt to explain the cumulative damage mechanism of bearing steels under RCF at the microstructure level. Fig. 1 shows the framework of this paper. In Section 2 we first establish a finite element model to obtain the distribution and variation rule of subsurface shear stress under bearing contact load. Then a two-phase atomic model of bcc-Fe and cementite is built and pretreated. Monotonic shear loads are carried out to obtain the mechanical properties of the model. Three types of shear loads are designed for cyclic deformation. In Section 3 we firstly analyze the shear stress responses under different loading conditions. Then the periodicity of statistical features, including dislocation density and proportion of irregular structures within the model, under cyclic loadings are discussed, respectively. Afterwards, the evolutions of dislocations under monotonic and cyclic shear loads are explained in detail. The morphology change of cementite phase after ten loading cycles are presented ultimately. The current work has partly explained the mechanism of cumulative damage initiation in bearing steels under RCF at the microstructure level.

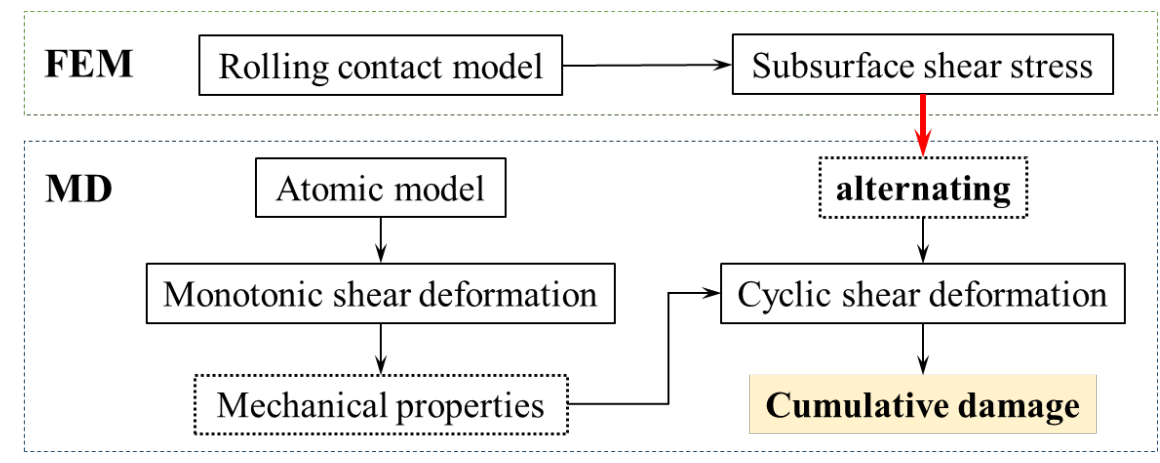


Fig. 1 Research framework of this paper. FEM, Finite element method. MD, Molecular dynamics.

\section{Methodology}

\subsection{Subsurface shear stress}

Most of the rolling contact fatigue failure originates from subsurface, so it is important to analyze the subsurface stress field during rolling contact. Note that the traditional Hertz contact theory is no longer applicable to subsurface stress analysis. Johnson ${ }^{26}$ calculated the principal stress at any depth under contact surface theoretically, and then used the Mohr circle to calculate shear stress. The subsurface shear stress reaches its maximum and minimum values at a certain depth, and changes alternately along the direction paralleling to bearing raceway. In this work, a two-dimensional (2D) finite element model was established to simulate the contact between ball and raceway. Due to the normal stress on subsurface is compressive, and compressive stress will hinder crack growth, the alternating shear stress is the key factor to result in damage accumulation and microcrack propagation. ${ }^{27}$ Therefore, only shear stress is used in the following research. Fig. 2 shows the distribution of shear stress in the contact area between ball and raceway. Ball has a diameter of $5.5 \mathrm{~mm}$ and inner race has a radius of $9.63 \mathrm{~mm}$ in the $2 \mathrm{D}$ contact model (shown in Fig. 2a). Contact analysis implemented in ABAQUS was used. Fig. 2b shows the nephogram of shear stress distribution in the contact area. It shows that the distribution of shear stress along the raceway has maximum and minimum values. Note that we are only interested in the stress distribution and ignore the specific stress value. Fig. 2c shows the maximum shear stress and the maximum orthogonal shear stress on the subsurface under contact load. The $x$-axis represents the direction along raceway, and $\tau_{\max }$ represents the maximum orthogonal shear stress which will change alternately as bearing runs. $\tau_{y}$ max stands for the maximum shear stress along the $y$-axis.

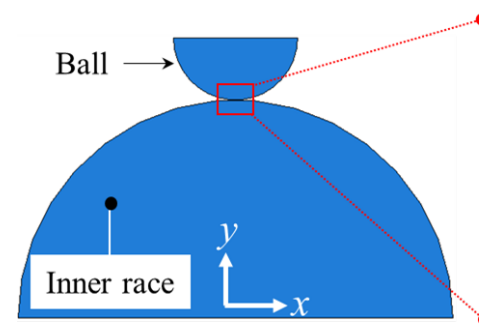

(a)

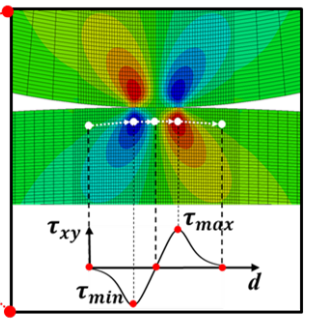

(b)

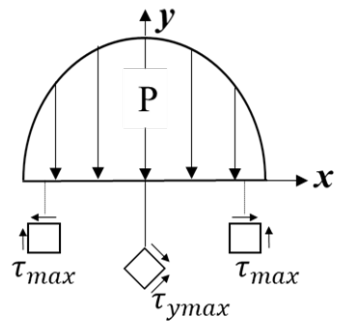

(c)

Fig. 2 Shear stress distribution in the contact area between ball and raceway. (a) Two-dimensional model of ball and raceway. (b) Nephogram of shear stress distribution in the contact area. (c) Maximum orthogonal shear stress and maximum shear stress on the subsurface under contact load.

According to the classical Hertz contact theory, the rolling contact between ball and raceway can be simplified to ideal line contact under 2D conditions (shown in Fig. 3). $p(x)$ represents the contact load distribution and $p_{\max }$ represents the maximum contact load which is specified $p_{\max }=2000 \mathrm{MPa} . p(x)$ is calculated by the following formula:

$$
p(d)=p_{\max }\left[1-(d / w)^{2}\right]^{1 / 2}
$$

Where $w$ represents the half-width of the contact area and is specified $w=0.25 \mathrm{~mm}$, and $d$ represents distance from the maximum load point. The computational domain is chosen to be $12 w$ length and $5 w$ depth with $0.01 w$ distance between each node horizontally or vertically (shown in Fig. 3a and b). $p(x$ ) is set to move uniformly along the $x$-axis by the DLoad subroutine implemented in ABAQUS, aiming to replace the rolling contact process between ball and raceway. Fig. $3 \mathrm{~b}$ presents the selected point P1 on the subsurface of the model. Fig. $3 \mathrm{c}$ shows the shear stress at point P1 as a function of loading time. The result 
is that shear stress at a certain point on the subsurface changes alternately with the motion of contact load. Considering the similarity between Fig. 3c and sine curve, the alternating shear stress is replaced by the stress with sinusoidal regularity in the interest of computational simplicity in the following sections.
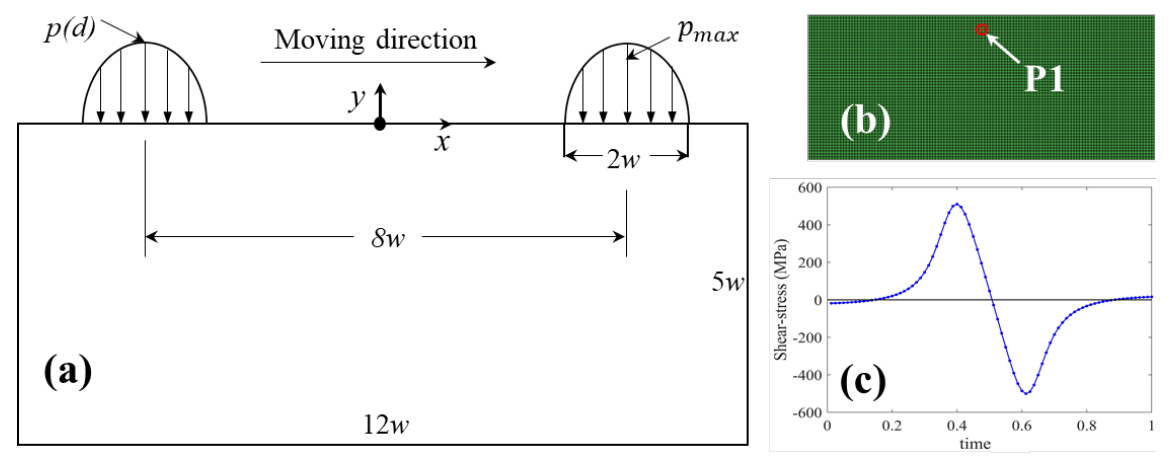

Fig. 3 Shear stress at a point on the subsurface under rolling contact load. (a) Dynamic Hertz contact load setting. (b) Position of node P1. (c) Change curve of shear stress at Point P1.

\subsection{Atomic model}

In order to study the characteristics of the interface between bcc-Fe and cementite, and to reveal the origin of cumulative damage, molecular dynamics (MD) simulations were performed using the Large-scale Atomic/Molecular Massively Parallel Simulator (LAMMPS) package. ${ }^{28}$ The MD model shown in Fig. 4 includes cementite phase in the middle and bcc-Fe matrix on both sides. The simulation model has a rectangular shape with dimensions $L_{x} \times L_{y} \times L_{z}=20 \times 20 \times 8.5 \mathrm{~nm}^{3}$ ( 0.3 million atoms). The upper and lower boundary layers will be applied loads along the $x$-axis or the $y$-axis to generate shear deformation of the atomic model. The length of the simulation model without boundary layers along the $z$-axis is specified $L_{z z}=7.4 \mathrm{~nm}$. The cementite inclusion has a length along the $y$-axis of $L_{y}^{c}=4.7 \mathrm{~nm}$. The bcc-Fe matrix has a calculated lattice parameter of $a_{0}=2.855 \AA$, and the lattice parameters of orthorhombic cementite are $a=5.088 \AA, b=6.670 \AA, c=4.470 \AA .^{29}$ The lattice orientation of the atomic model can be expressed as $x:[111]_{\alpha}\left\|[100]_{c}, y:[112]_{\alpha}\right\|[010]_{c}$ and $z:[110]_{\alpha} \|[001]_{c}$. Here the subscripts $a$ and $c$ denote bcc-Fe and cementite, respectively. The widely accepted Bagaryatskii orientation relation between ferrite and cementite was referred and adopted here. ${ }^{24,30}$ The interatomic potential of $\mathrm{Fe}-\mathrm{C}$ proposed by Liyanage et al. ${ }^{31}$, which is based on a modified embedded atom method (MEAM), was adopted to describe the interatomic force. The potential was proved to have high precision in MD annealing and simulation. ${ }^{23}$ Periodic boundary conditions were applied in all three directions to reduce the size limitations of the MD simulation. Molecular statics energy minimization was carried out at zero pressure and $0 \mathrm{~K}$ by using the conjugate gradient algorithm. Then MD annealing was performed. The Nosé-Hoover isothermal-isobaric (NPT) ensemble ${ }^{32-34}$ was used to the simulation system to assign a temperature of $800 \mathrm{~K}$ and further equilibrate thermally at $800 \mathrm{~K}$ for 100 ps. After that, the system was cooled down to $5 \mathrm{~K}$ within $50 \mathrm{ps}$. The temperature of $5 \mathrm{~K}$ was chosen to better observe the interface reactions caused by shear motion rather than being dominated by temperature. 


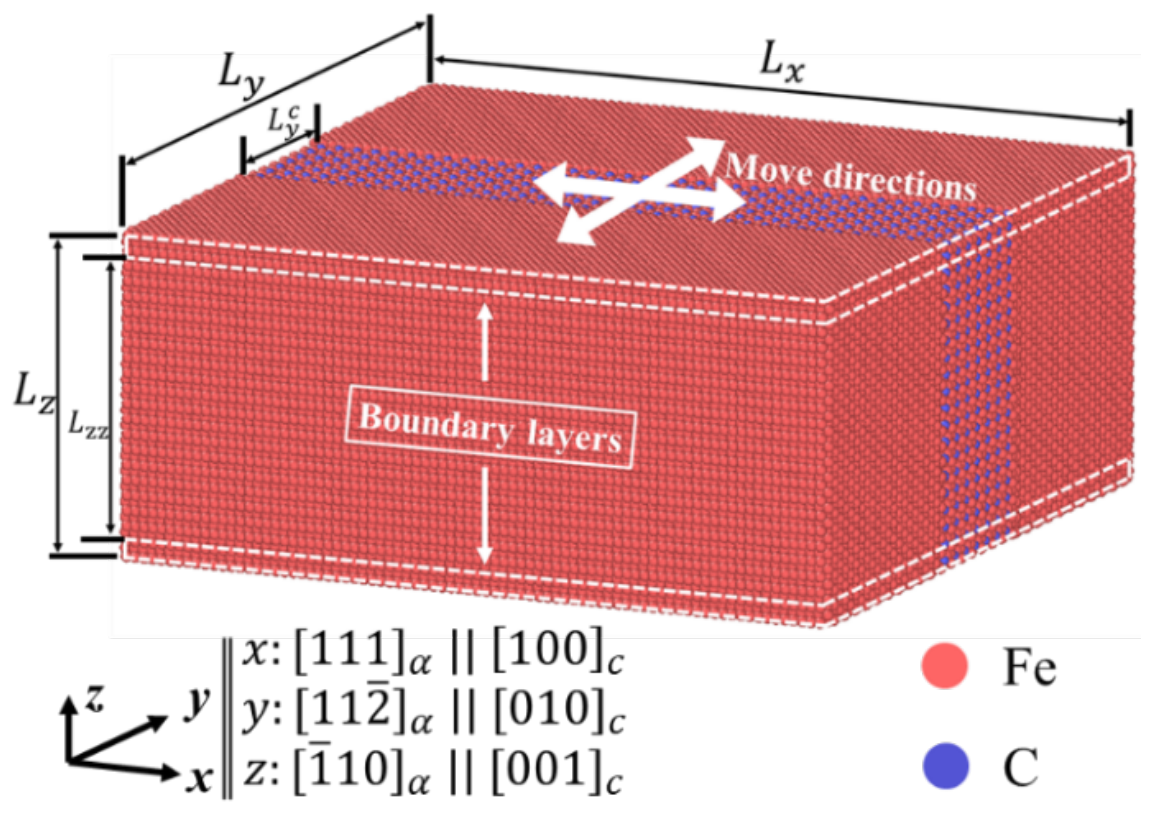

Fig. 4 Atomic model of bcc-Fe and cementite, in which $\alpha$ and c represent $\alpha$-Fe and cementite, respectively. Red and blue atoms represent iron and carbon atoms, respectively. White dotted boxes refer to boundary layers.

\subsection{Loading conditions}

Displacement loads were applied to the boundary layers of the atomic model to form alternating shear stress conditions. Because of the deformation properties of bearing steels, it is necessary to discuss the transition between elasticity and plasticity of the atomic model under shear deformation. Therefore, monotonic displacement loads were performed to discuss the transition (shown in Fig. 5), which were useful for establishing the load levels to be applied under cyclic deformation. Displacement loads at a constant velocity of $1 \AA / p s$ were applied to the upper and lower boundary layers of the simulation model in opposite directions (shown in Fig. 5a). The MD timestep was $2 \mathrm{fs}$, and the temperature was maintained at 5K. Here $d$ denotes the displacement difference between the upper and lower boundary layers. $\gamma$ represents the shear strain of the model and is calculated by $d$ and $L_{z z}$. Considering that loading directions will affect the simulation results, the movement of the boundary layers is set along the $y$-axis and the $x$-axis, respectively.

Fig. 5b shows the shear stress- $d$ curves under monotonic loads along two directions. Note that the shear stress refers to the average values calculated along the $y$-axis $\left(\tau_{\mathrm{yz}}\right)$ and the $x$-axis $\left(\tau_{\mathrm{xz}}\right)$ of the model except for the boundary layers, ${ }^{35}$ respectively. The linear shear stress tensor-yz and linear shear stress tensor-xz shown in Fig. 5 denote the results of loading along the $y$-axis and the $x$-axis, respectively. Although the movement of the boundary layers along the two directions resulted in different stress states for the model, both cases contain three different stages: elastic, elastic-plastic and plastic. Note that the initial stresses of the shear stress- $d$ curves in Fig. $5 \mathrm{~b}$ are not zero. This is because that the MD annealing only kept the normal stress tensors in three directions at zero, but not shear stress tensors. However, due to these initial shear stresses are far less than the stress of interest, the influence of them on the subsequent simulation results can be ignored. 

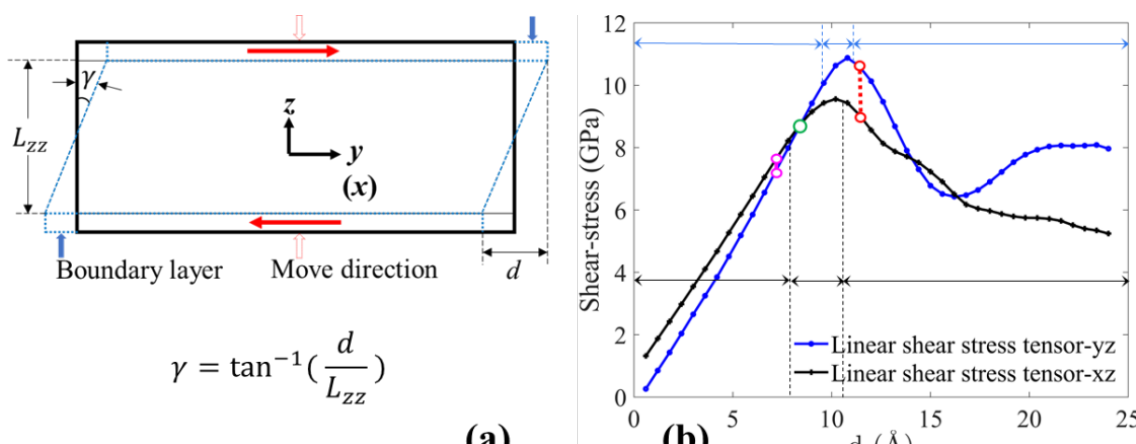

(a)
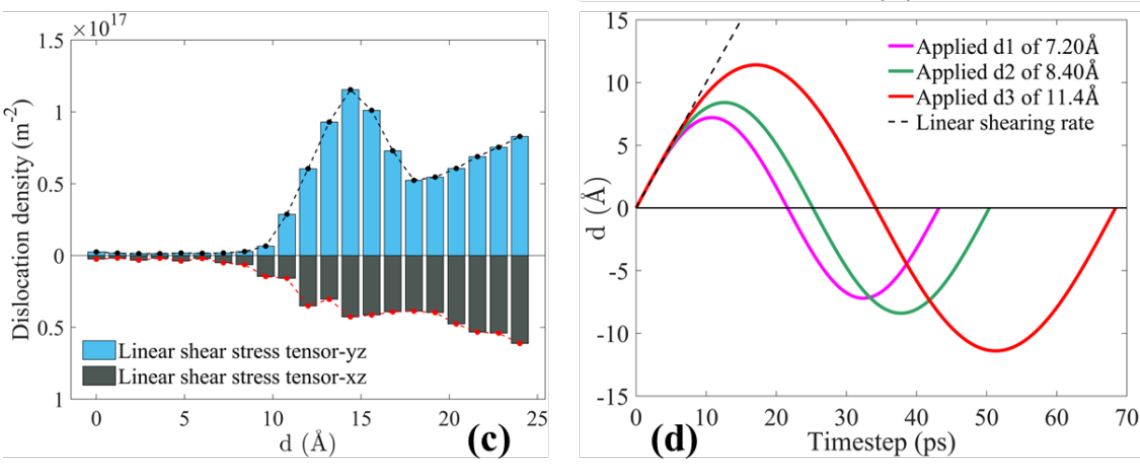

Fig. 5 Shear stress and dislocation density of the model under monotonic loads and the cyclic load settings of the model. (a) Motion diagram of the boundary layers and the calculation formula of shear strain. (b) Shear Stress-d curves under monotonic loads. Linear shear stress tensor-yz and linear shear stress tensor-xz represent the results of loading along the $y$-axis and the $x$-axis, respectively. Open symbols indicate the designed cyclic load levels. Both curves are respectively divided into three stages: elastic, elastic-plastic and plastic. (c) Dislocation density-d bars under monotonic loads. (d) d-time curves for cyclic deformation with different period and load amplitude. Periods are 43.2ps (d1), 50.4ps (d2) and 68.4ps (d3).

Statistical results of dislocation density within the model are shown in Fig. 5c. Dislocation density formula is written as (unit: $\left.m^{-2}\right)$ :

$$
\rho=\frac{L}{V}
$$

Where $\rho$ is the total length of dislocation lines in the crystal per unit volume, $L$ is the total dislocation length, and $V$ is the volume of crystal. Fig. 5c presents that only a few dislocations exist in the model when $d$ is less than $10 \AA$. Then the dislocation densities both increase rapidly when the model is transformed into the elastic-plastic stage and remain high in the plastic stage. These two types of dislocation density are distinguished by the loading directions and affected by the lattice orientation. ${ }^{36}$

To explore the elastic-plastic transition characteristics and plastic accumulation principle of the simulation model, we selected three shear stress states for the following cyclic simulations as shown in Fig. 5b. The three different displacement loads named d1, d2 and d3 were applied values of $7.2 \AA, 8.4 \AA$ and $11.4 \AA$, respectively (shown in Fig. 5d). According to the formula of $\gamma($ shown in Fig. 5a), the shear strains corresponding to d1, $\mathrm{d} 2$ and $\mathrm{d} 3$ are $0.097 \mathrm{rad}, 0.113 \mathrm{rad}$ and $0.153 \mathrm{rad}$, respectively. All the three load curves shown in Fig. $5 \mathrm{~d}$ are sinusoidal curves, and the initial velocities of the cyclic loads are similar with the velocity of the monotonic load above. As shown in Fig. 5b and d, the model is in the elastic stage when the load is applied along the $y$-axis and the $x$-axis under $\mathrm{d} 1$. When the load is $\mathrm{d} 2$, the model is in the elastic stage under load along the $y$-axis and is in the elastic-plastic stage under load along the $x$-axis. When the load goes up to d3, the 
model is in the plastic stage along both loading directions. Actually, the variation of the alternating shear stress on the subsurface during RCF is similar to the impulse waveform. ${ }^{25}$ The alternating shear stress in a certain region is close to zero in the most time of a cycle. However, we ignored the period when the model was at a low alternating shear stress level to reduce the simulation cost. The cyclic shear movement of the boundary layers was set to be continuous. Fig. 5d shows only one load cycle, and we conducted 10 cycles for all three load amplitudes to explore the plastic cumulative damage mechanism of the model. The OVITO software package ${ }^{37}$ was used to visualize the different defects in the system. Dislocations were analyzed by using the dislocation extraction algorithm (DXA) tool. ${ }^{38}$ Common neighbor analysis (CNA) ${ }^{39,40}$ was used to classify atoms in the crystalline structure.

\section{Results and Discussion}

\subsection{Shear stress responses}

Cyclic shear stress was calculated to investigate the plastic accumulation behavior of the simulation model. The cyclic loads as shown in Fig. 5d were applied to the boundary layers along the $y$-axis and the $x$-axis, respectively. Fig. 6a and d show the curves of the shear stress as functions of cycle when $\mathrm{d} 1$ is applied along the $y$-axis and the $x$-axis, respectively. In the first three cycles, the maximum stress amplitudes remain unchanged in both curves, indicating that the model is in the elastic stage. Then the stress amplitudes decrease to some extent from the fourth cycle. The decrease is called cyclic softening, ${ }^{41}$ which also stands for the beginning of plastic accumulation. Although the model is in the elastic stage when the load is d1 (shown in Fig. 5b), dislocations nucleate and grow on the interface between cementite and bcc-Fe matrix, thus resulting in plastic accumulation. The details will be discussed in the following sections. Fig. 6b and e show the curves of the shear stress as functions of cycle when $\mathrm{d} 2$ is applied along the $y$-axis and the $x$ -axis, respectively. Cyclic softening occurs after the first cycle or in the first cycle due to the diverse loading directions. Fig .6c and $\mathrm{f}$ show that when the load rises to $\mathrm{d} 3$, shear stress amplitudes decrease in the first cycle and then reach a steady level. The conclusion is that larger load leads to earlier cyclic softening. In addition, the red boxes in Fig .6c and $\mathrm{f}$ show that the shear stress yields obviously when the load is large enough, and large residual shear stress exists within the model at the end of 10 cycles. Fig. 6 also shows that the variational tendencies of shear stress $\tau_{\mathrm{yz}}$ and $\tau_{\mathrm{xz}}$ are basically similar under the same loading amplitude.
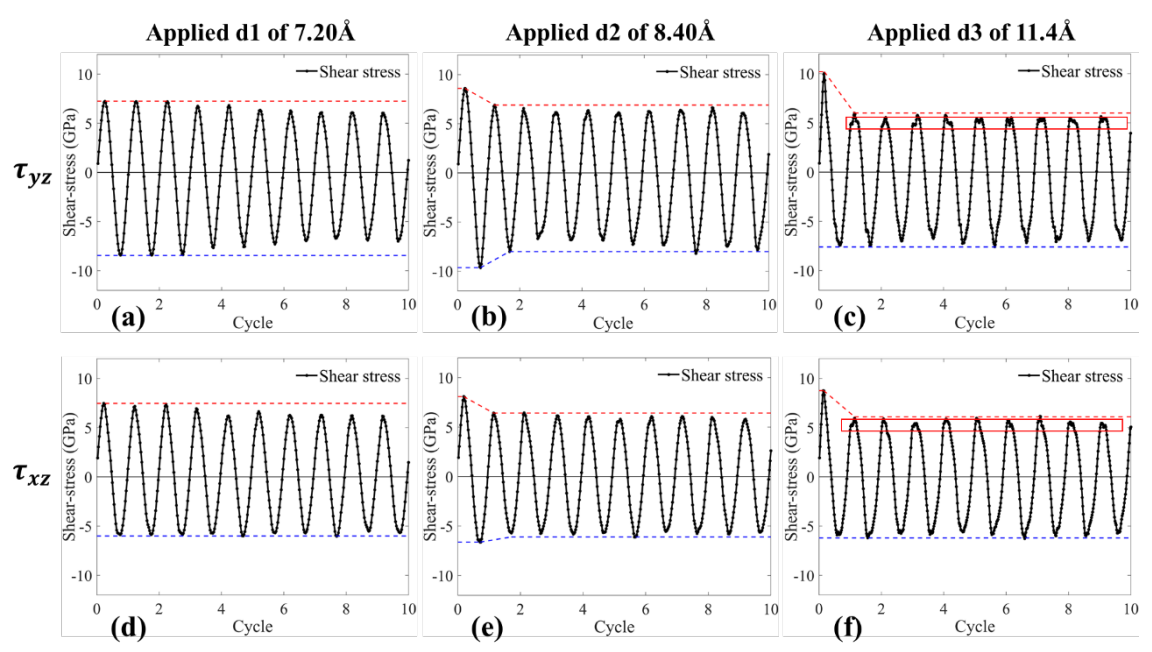

Fig. 6 Shear stress-cycle responses under different loading conditions. Applied loads of (a, d) d1, (b, e) d2 and $(\mathrm{c}, \mathrm{f}) \mathrm{d}$. Loading directions are along $(\mathrm{a}, \mathrm{b}, \mathrm{c})$ the $y$-axis and $(\mathrm{d}, \mathrm{e}, \mathrm{f})$ the $x$-axis, respectively. Red and blue dash lines represent the trends of maximum and minimum stress amplitudes, respectively. Red boxes show the yields of shear stress. 
The decreasing extent of stress amplitude during cyclic softening depends on the dislocation evolution mechanism under different shear loads. Note that the experimental results drawn from carbon steels show work hardening as cyclic cycles increase under uniaxial ratchetting deformation, ${ }^{11,42}$ which is contrary to the cyclic softening. The reason might derive from the initial regularity and simplification of our simulation model. Furthermore, there are obvious disparities between the results shown in Fig. 6 and the results drawn from the model under cyclic tension and compression loadings. ${ }^{20}$ This is mainly due to the differences in the calculated stress tensors and the mode of motion. During cyclic shear deformation, atomic model seems more likely to appear cyclic softening, and the plastic accumulation is faster. And the rapid plastic accumulation is unfavorable to the fatigue life of bearing steels.

\subsection{Periodicity of statistical features}

Cyclic fatigue involves in plastic deformation caused by dislocation nucleation and movement. ${ }^{43}$ Fig. 7 shows the statistical results of dislocation density in the model under cyclic loads. Note that only the bcc-Fe matrix is capable of producing identifiable dislocations due to the complex crystal structure of cementite. ${ }^{44}$ The upper and lower parts of Fig. 7a show the variation of dislocation density with cyclic cycles when d1 is applied along the $y$-axis and the $x$-axis, respectively. When the load is along the $y$-axis, the dislocation density in the first three cycles approaches to zero, corresponding to the elastic deformation process in the first 3 cycles in Fig. 6a. Then the dislocation density increases obviously in the fourth cycle and remains at a high level after that. Note that the increase of dislocation density generally represents the elastic-plastic transition process of the model, which also explains the cyclic softening in Fig. 6a. In addition to that, large amounts of dislocations mean plenty of residual shear strain in the model. When the load is along the $x$-axis, the dislocation density rises to relatively large values during the first three cycles but falls to close to zero at the end of each cycle (shown in Fig. 7a). This could be corresponding to the nucleation and annihilation process of dislocations in the model. The dislocation annihilation at the end of each cycle might also be the reason why the shear stress peaks in the first three cycles in Fig. 6d hardly change. After the third cycle, the dislocation density stabilizes at a high level due to the dislocation annihilation being hindered, which is also the reason for the cyclic softening in Fig. 6d.

The upper and lower parts of Fig. 7b show the variation of dislocation density with cyclic cycles when $\mathrm{d} 2$ is applied along the $y$-axis and the $x$-axis, respectively. The dislocation density increases significantly after the first cycle under both loading directions, which is corresponding to the results shown in Fig. $6 \mathrm{~b}$ and e. High dislocation density after the first cycle led to cyclic softening. The upper and lower parts of Fig. 7c show the variation of dislocation density with cyclic cycles when $\mathrm{d} 3$ is applied along the $y$-axis and the $x$ -axis, respectively. The model is in the plastic stage under $\mathrm{d} 3$ as shown in Fig. 5b. The dislocation density has stabilized at a high level since the first stage, which may be the main reason for the yields of shear stress in Fig. 6c and f. Furthermore, the average value of dislocation density increases with the rise of the load and almost has no relation with the direction of the load. 

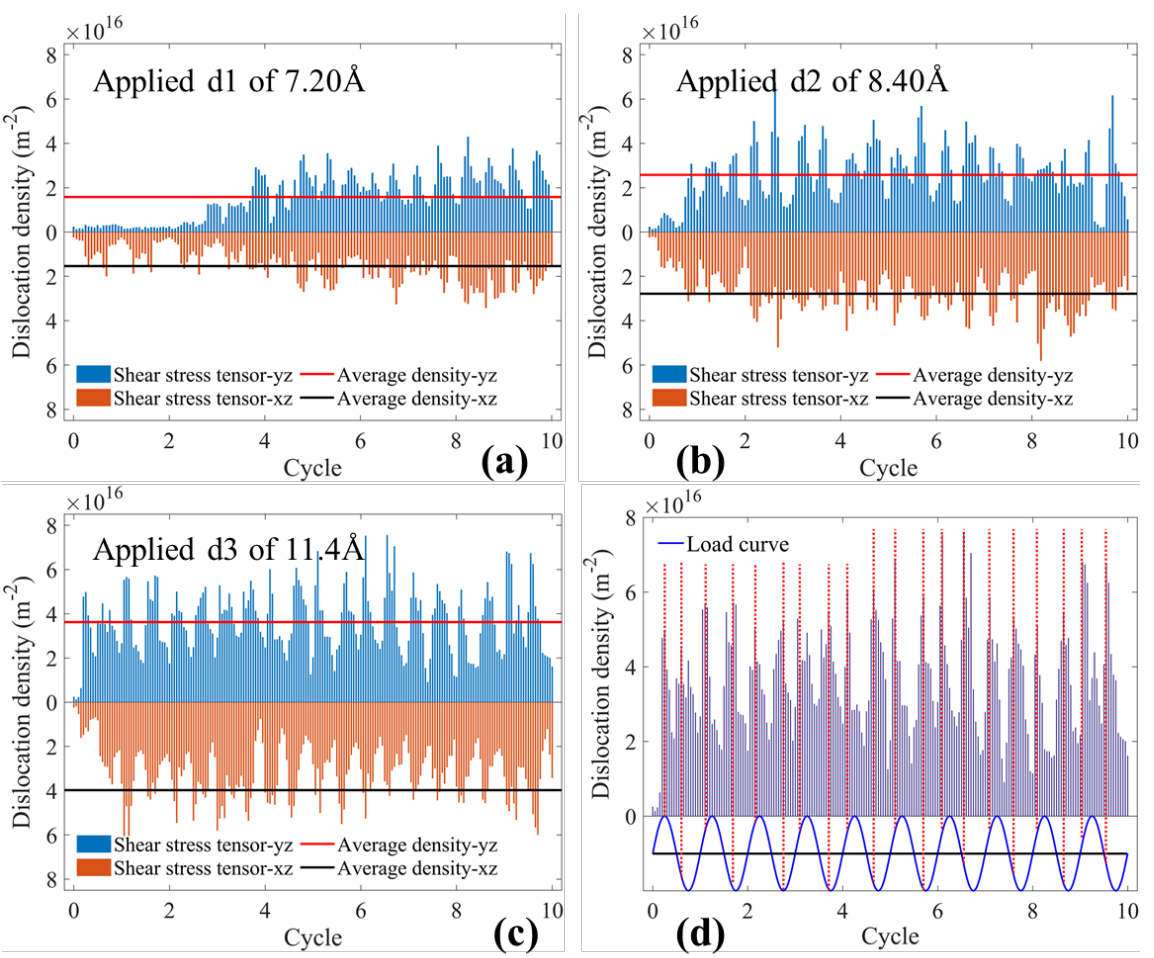

Fig. 7 Evolution of dislocation density under different cyclic loads. (a), (b) and (c) show the evolutions of dislocation density under $\mathrm{d} 1, \mathrm{~d} 2$ and $\mathrm{d} 3$, respectively. Shear stress tensor-yz and shear stress tensor-xz represent the results of loading along the $y$-axis and the $x$-axis, respectively. Average density-yz and average density-xz represent the average values of dislocation density when loads are applied along the $y$ -axis and the $x$-axis, respectively. (d) Periodic variation of dislocation density. Red dotted lines indicate the two peaks of dislocation density in each load cycle.

The elasticity of the model promotes dislocation annihilation, while the plasticity of the model promotes the overgrowth of dislocation. Therefore, the evolution of dislocation density is another manifestation of the elastic-plastic properties of the model. In addition to that, the variation of dislocation density is closely related to the cyclicity of applied loads. Fig. 7d shows a detailed description of the upper part of Fig. 7c. The blue curve at the bottom of Fig. 7d shows the variation of cyclic load with cyclic cycles. The red dotted lines indicate the two peaks of dislocation density in each cycle. The variation of dislocation density is highly periodic and corresponds to the leading edges of the load peaks in the blue curve below. When the load goes to zero, the dislocation density appears to be a minimum. And the minimal values within each cycle are greater than zero, which indicates the plastic accumulation in the model. Large amounts of line defects and concomitant point defects in the model will affect the macroscopic plasticity of the material. When the microscopic defects develop into macroscopic cracks or holes with the motion of shear slip bands in the model, the fatigue life of the material will be adversely affected. 

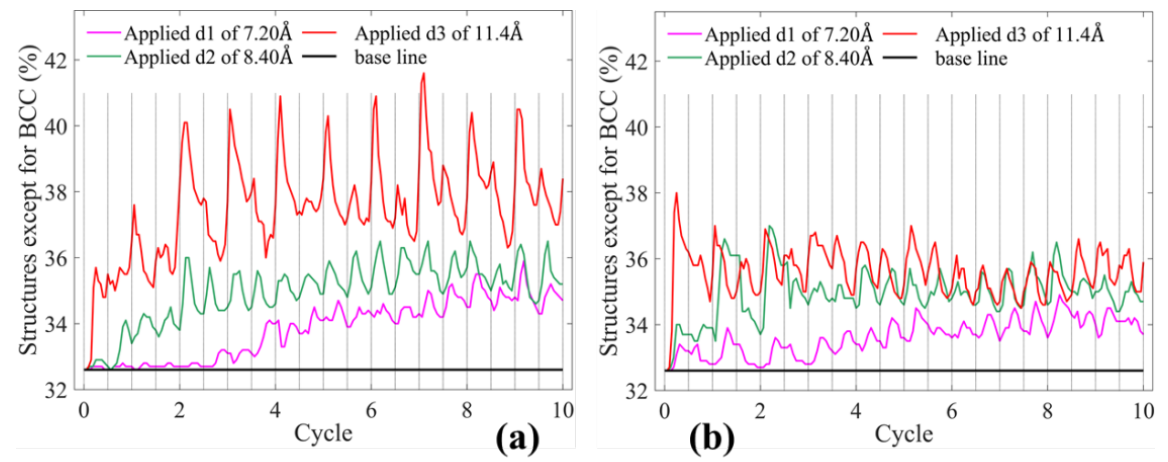

Fig. 8 Proportion of irregular structures in the model. Loading directions are along (a) the $y$-axis and (b) the $x$-axis, respectively. Magenta, green and red lines refer to $\mathrm{d} 1, \mathrm{~d} 2$ and $\mathrm{d} 3$, respectively. Base line refers to the proportion of cementite phase.

Plastic deformation of materials generally means that regular structures tend to be chaotic, and it also corresponds to the increase in the proportion of irregular structures. Statistical analysis of the proportion of irregular structures within the model can explain the mechanism of plastic deformation to some extent. ${ }^{45}$ The bcc-Fe matrix in the atomic model can be identified as regular BCC structure by CNA algorithm, while cementite phase, point defects and surface defects such as stacking faults are identified as irregular structures. Fig. 8 shows the statistical results of the proportion of irregular structures in the model under cyclic loads. The loading directions in Fig. $8 \mathrm{a}$ and $\mathrm{b}$ are along the $y$-axis and the $x$-axis, respectively. The base line in Fig. 8 represents the proportion of cementite phase in the model. Some of the same variation rules can be drawn from Fig. 8a and b. When the load is d1, the proportion of the irregular structures in the first three periods is close to the base line and then remains at a high level. When the load is $\mathrm{d} 2$, the proportion of irregular structures gradually increases after the first period and then remains at a high level. The proportion of irregular structures is always kept at a high level under d3. And all the curves shown in Fig. 8 have obvious periodicity. The above rules are consistent with the variation rules of dislocation density drawn from Fig. 7. However, when the load is $\mathrm{d} 3$, the average value and amplitude fluctuations of the proportion of irregular structures shown in Fig. 8a are larger than these shown in Fig. 8b, which is different from the conclusion drawn from Fig. 7. A relatively reasonable explanation is that different loading directions correspond to different mechanisms of dislocation nucleation and annihilation, and this will be discussed in detail in the following sections.

\subsection{Evolution of dislocations}

Dislocation is a kind of line defect in crystal, which plays a decisive role in the plastic deformation, strength and fracture of materials. ${ }^{46}$ Fig. 9 shows the details of dislocation evolution under monotonic loads. Fig. 9a and $\mathrm{b}$ show the dislocation distribution when loads are applied along the $y$-axis and the $x$-axis, respectively. Fig. 9c provides the initial structure for comparison. In Fig. 9a, dislocations begin to nucleate from the interface between cementite and bcc-Fe when the load reaches $\mathrm{d} 3$, and then grow with the increase of shear load. Under periodic boundary conditions, dislocations gradually connect the left and the right interfaces, which manifests as dislocations initiating from one interface and growing through the boundary to the other interface. This indicates that dislocations cannot annihilate and continue to remain in the material, marking the beginning of plastic accumulation of the material. Fig. 9b shows that with the increase of shear load, the quantity of dislocation gradually increases, and the more significant feature is the appearance of defect meshes near the interface. Defect mesh is a triangulated mesh which represents the bad crystal regions that have not been classified as dislocations. ${ }^{37,47}$ In the present work, it represents the residual shear strain in the bcc-Fe matrix due to cyclic loading, and further indicates the residual shear stress in the model. The appearance of defect mesh corresponds to the increase of local micro-plastic flow and residual stress of the material in stage I of RCF, which is not conducive to the fatigue life of the bearing. ${ }^{11}$ Note that when the 
monotonic load applied along the $x$-axis reaches $24.0 \AA$, the defect meshes on the left and right interfaces have the same orientation, as shown in the red dashed boxes in Fig. 9b. This pattern usually indicates the presence of large shear slip bands in the model. Meantime, it also refers to the transfer of the slip bands from bcc-Fe matrix to cementite phase, indicating that shear deformation begins to occur inside or on the surface of the cementite phase. When shear slip occurs in the brittle cementite phase, the fatigue failure of the material begins to develop.

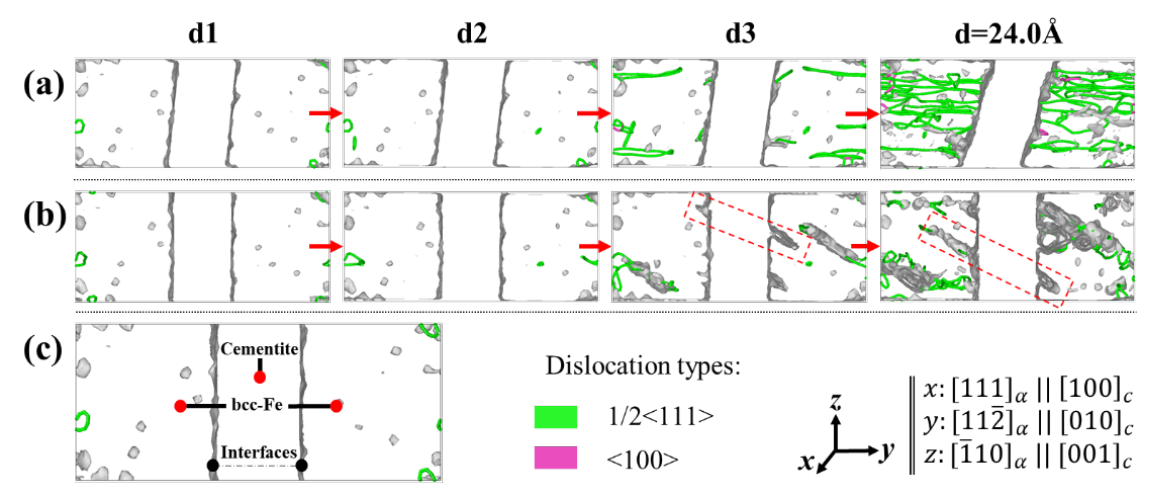

Fig. 9 Dislocation distribution in the model under monotonic loads. Loading directions are along (a) the $y$-axis and (b) thex-axis, respectively. The initial structure is provided for comparison in (c). Green and magenta lines in bcc-Fe matrix correspond to $1 / 2<111>$ and $<100>$ dislocations, respectively. Gray planes in bcc-Fe matrix are defect meshes. Red dashed boxes contain defect meshes with the same orientation.

Cyclic softening and elastic-plastic transformation in Fig. 6 are closely related to the evolution of dislocations during cyclic shear deformation. Fig. 10 shows the dislocation distribution in the model underlying cyclic deformation. Fig. 10a-c correspond to the loading conditions of $\mathrm{d} 1$, $\mathrm{d} 2$ and $\mathrm{d} 3 \mathrm{respectively,} \mathrm{and} \mathrm{the} \mathrm{loading}$ direction is along the $y$-axis. Fig. 10a shows that dislocations begin to appear at the end of the third cycle, and then increase and remain at a low level in the following cycles. Fig. 10b shows that dislocations begin to appear at the end of the first cycle. When the load is $\mathrm{d} 3$, a large amount of dislocations and defect meshes exist in the bcc-Fe phase at the end of the first cycle. The proportion of irregular structures is large, and the quantity of dislocations and defect meshes remains high throughout the whole cyclic loadings. The dislocation evolution under these three conditions corresponds to the variation of dislocation density shown in Fig. 7. The defect meshes shown in the red dashed boxes in Fig. 10c have the same orientation, which is similar with the results shown in Fig. 9b, indicating that there exists a lot of residual strain in the model. This also points out that the material has undergone severe plastic deformation and damage accumulation, and the cementite phase tends to be damaged. ${ }^{20}$ Note that there are more defect meshes in Fig. 10c than those in Fig. 9a, indicating that cyclic shear deformation is more destructive to the structure than monotonic shear deformation, and is more likely to result in fatigue failure of the material.

Fig. 10d-f correspond to the loading conditions of $\mathrm{d} 1, \mathrm{~d} 2$ and $\mathrm{d} 3$ respectively, and the loading direction is along the $x$-axis. When the load is $\mathrm{d} 1$, the interfaces are free of dislocations at the end of the third cycle, after which the dislocations increase and remain at a low level. When the load is $\mathrm{d} 2$, dislocations begin to appear at the end of the first cycle. It is noted that under these two loading conditions, most of the dislocations in the model are annular, germinating from the interface and eventually remaining in the bcc-Fe phase. When the load reaches $\mathrm{d} 3$, dislocations and obvious defect meshes are generated at the end of the first cycle. In the subsequent cycles, although there are still many dislocations in the model, no obvious defect mesh could be found on the interface. The less residual shear strain in the model can be attributed to that the direction of the cyclic loadings is parallel to the interface. This indicates that the model produces less plastic accumulation, or the rate of plastic accumulation is slower, which may result in a relatively longer fatigue life of the material. It is worth noting that, under cyclic deformation, fewer defect meshes are 
generated in the model when loads are along the $x$-axis, which is contrary to the rule drawn from Fig. 9 . This suggests that the plastic accumulation mechanism of the material is ascribed to the synergistic effects between the cyclic deformation and the loading direction.

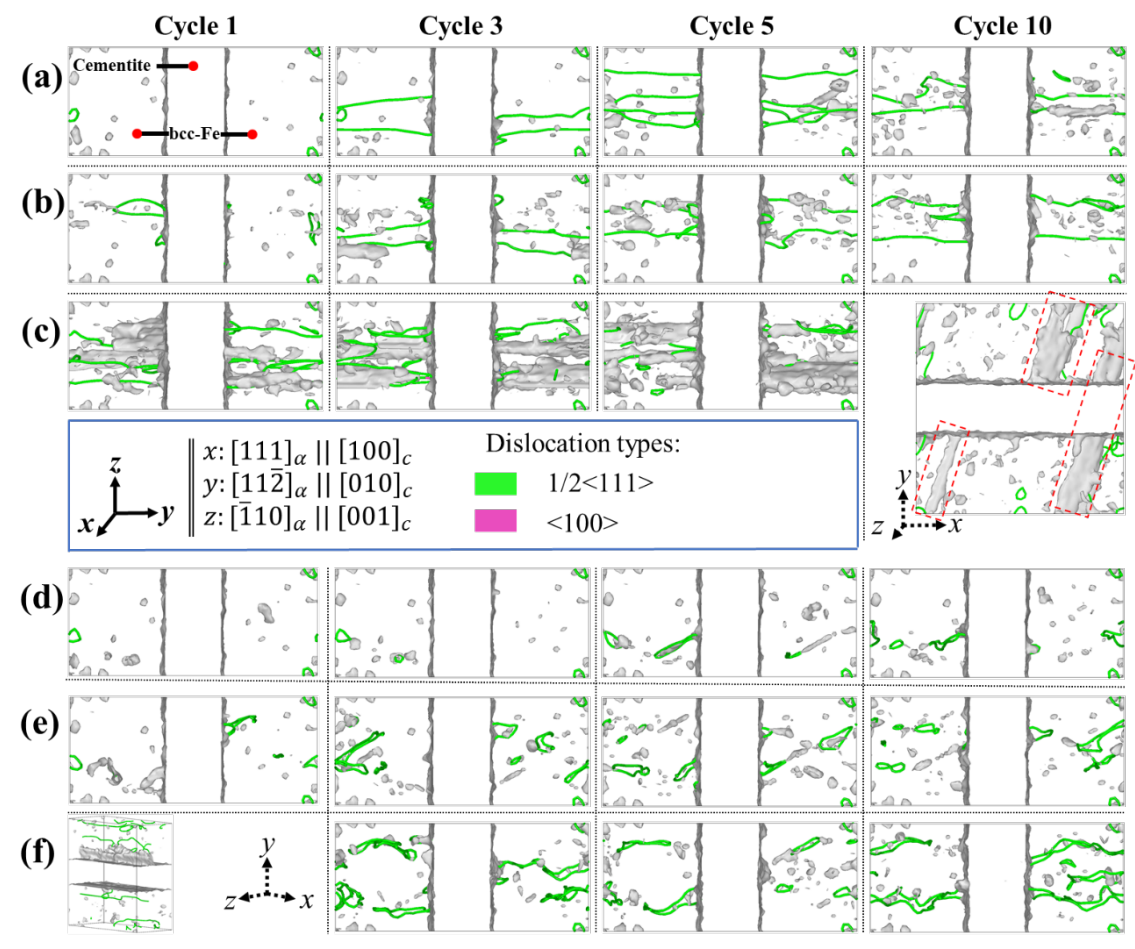

Fig. 10 Dislocation distribution in the model under cyclic loads. Applied loads of (a, d) d1, (b, e) d2 and $(\mathrm{c}, \mathrm{f}) \mathrm{d} 3$. Loading directions are along $(\mathrm{a}, \mathrm{b}, \mathrm{c})$ the $y$-axis and $(\mathrm{d}, \mathrm{e}, \mathrm{f})$ the $x$-axis, respectively. Only dislocation distribution at the end of the cycle $(1,3,5,10)$ is shown. Other features are the same as in Fig. 9 .

The interface is an underlying area for defect generation and annihilation during plastic deformation. ${ }^{48}$ During cyclic shear deformation, the interface is accompanied by the nucleation and annihilation of dislocations, which results in the fluctuation of dislocation density (shown in Fig. 7). After several cycles, the residual strain in the model hinders the annihilation of dislocations, which is manifested as a large number of dislocations remaining in the model at the end of the cycle. Fig. 11 shows the nucleation and annihilation of dislocations on the interface when $\mathrm{d} 1$ is applied along the $y$-axis. The view in Fig. 11 is kept parallel to the interface by rotating the coordinate system. In the third cycle shown in the upper part of Fig. 7a, the dislocation density first increases and then decreases, which is manifested by the evolution of the dislocation as shown in Fig. 11. The $1 / 2<111>$ dislocation nucleates at 2.25 cycles, then gradually grows up at 2.25 2.4375 cycles, and finally disappears at $2.4375-2.5625$ cycles. Note that the time for dislocation nucleation is longer than that for annihilation, which is mainly due to a larger activation barrier of nucleation than that of annihilation. The same conclusion was drawn by Liang et al. ${ }^{20}$ In addition to that, low temperature was adopted to the atomic model, because temperature affects the speed of dislocation evolution. ${ }^{49}$ Choosing a temperature of $5 \mathrm{~K}$ could help us observe the process of dislocation nucleation and annihilation more clearly. 


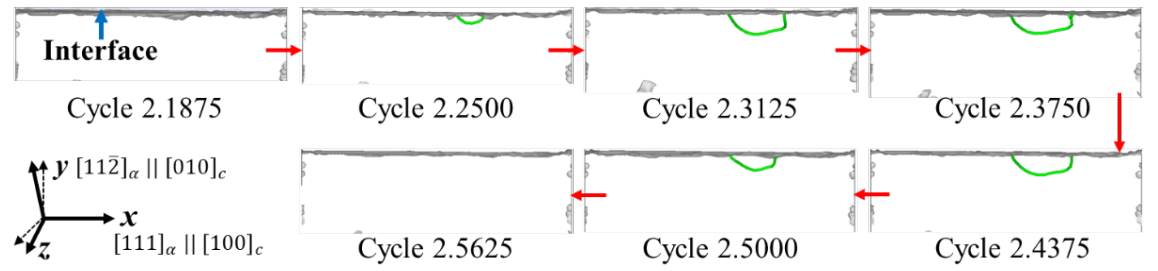

Fig. 11 Nucleation and annihilation process of a $1 / 2<111>$ dislocation on the interface when $\mathrm{d} 1$ is applied along the $y$-axis. The whole process is contained in the third cycle. The view is kept parallel to the interface by rotating the coordinate system.

Figure 12 shows the growth process of dislocations on the interface when $\mathrm{d} 3$ is applied along the $y$ - axis. The whole process took place within the first quarter of the first cycle, from 11.2ps to 13.6ps (shown in Fig. 5d). With the increase of shear deformation, a large number of dislocations begin to nucleate and grow rapidly (shown in Fig. 12), eventually leading to a dislocation density peak in Fig. 7c. In addition, the large residual strain causes the overgrowth and reaction of dislocations. During the reduction of shear deformation, lots of dislocations still remain in the model, which is the main reason for the large number of dislocations and defect meshes in the model at the end of the first cycle in Fig. 10c.

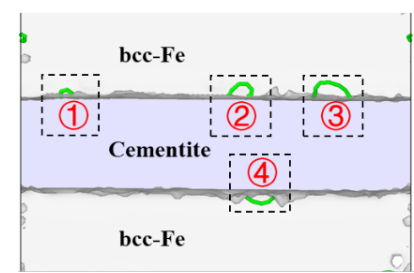

(a)

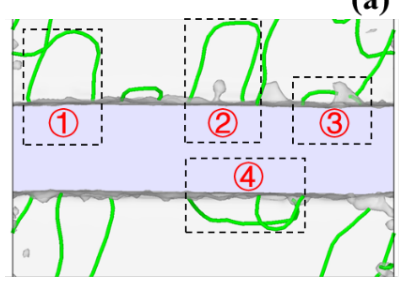

(c)

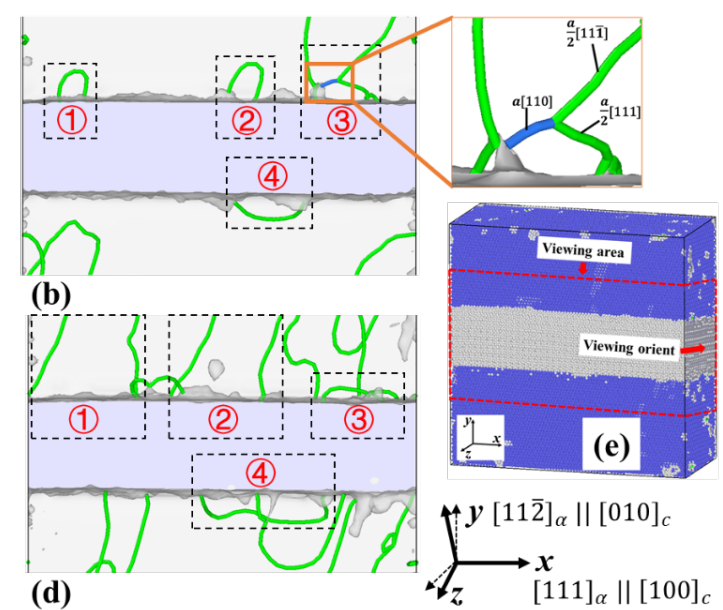

(d)

Fig. 12 Growth process of dislocations on the interface when $\mathrm{d} 3$ is applied along the $y$-axis. The whole process is contained in the first quarter of the first cycle. (a) timestep $=11.2 \mathrm{ps}, \mathrm{d}=9.766 \AA$. (b) timestep $=12.0 \mathrm{ps}$, $\mathrm{d}=10.172 \AA$. (c) timestep $=12.8 \mathrm{ps}, \mathrm{d}=10.522 \AA$. (d) timestep $=13.6 \mathrm{ps}, \mathrm{d}=10.816 \AA$. Green and blue lines in bcc-Fe matrix correspond to $1 / 2<111>$ and $<110>$ dislocations, respectively. (e) Viewing area and orient of the dislocation growth. The view is kept parallel to the interface by rotating the coordinate system.

Severe shear deformation leads to excessive growth of dislocations which evolve into surface defects such as stacking fault, and the interlaced dislocations ultimately result in irreversible plastic accumulation. Note that the nucleation of dislocations usually occurs on the interface between bcc-Fe and cementite. Therefore, it is convincing that the generation and accumulation of dislocations on the two-phase interface or the boundary of carbide inclusions are the origin of the cyclic plastic accumulation. This may also be the main reason why bearing steels damage usually appears near inclusions such as carbides.

\subsection{Morphology of cementite phase}

The present work has shown that although cementite is much harder than matrix in bearing steels, it still participates in shear deformation. Fig. 13 shows the morphologies of the cementite phase under different 
loading conditions after ten cycles. As the load applied along the $y$-axis increases, the surface morphology of the cementite phase is destroyed more seriously, and the regular lattice structure tends to be chaotic (shown in Fig.13a-c). When the load applied along the $x$-axis increases, the volume of the cementite phase change inconspicuously (shown in Fig.13d-f). This is due to the fact that the loading direction is parallel to the interface of the two phases. Nevertheless, the regular crystal structure of the cementite phase is destructed and tends to be disordered when the shear deformation is large enough as shown in Fig. 13f. The above description is consistent with the mechanism of dislocation evolution drawn from Fig. 10. The residual shear strain in the model cumulates, as the shear load increases, and the cementite phase prefer to be damaged.

Plastic deformation results in dislocations initiating and growing from one interface to another until they are absorbed by the cementite phase. And the absorbed dislocations may then issue in a local shear deformation in the cementite phase. ${ }^{23}$ In addition to that, when the shear zone in bcc-Fe matrix is thick enough to penetrate the interface, the shear zone is formed in the cementite phase. It is known that a breaking of brittle phase such as cementite works as a trigger of cleavage fracture initiation. ${ }^{50}$ Under the joint induction of RCF and the inclusion defects within the subsurface, severe cyclic shear deformation will destroy brittle phase such as cementite inclusion in bearing steels, and thus impair the fatigue life of bearing.

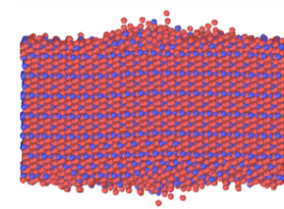

(a)

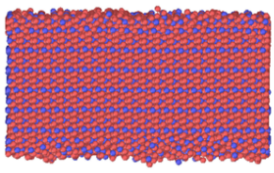

(d)

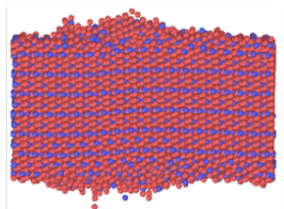

(b)

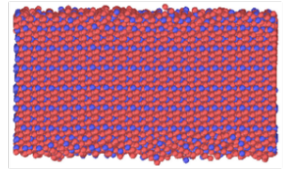

(e)

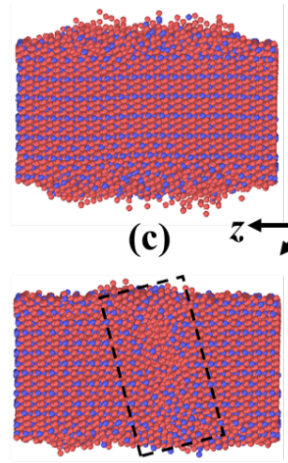

(f)

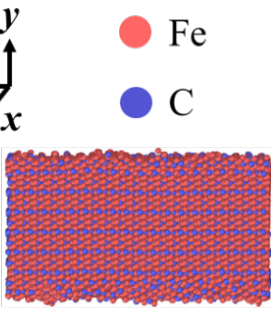

(g)

Fig. 13 Morphologies of the cementite phase under different loading conditions after ten cycles. Applied loads of $(\mathrm{a}, \mathrm{d}) \mathrm{d} 1,(\mathrm{~b}, \mathrm{e}) \mathrm{d} 2$ and $(\mathrm{c}, \mathrm{f}) \mathrm{d}$ 3. Loading directions are along $(\mathrm{a}, \mathrm{b}, \mathrm{c})$ the $y$-axis and $(\mathrm{d}, \mathrm{e}, \mathrm{f})$ the $x$-axis, respectively. Black dashed box shows the damaged morphology of the cementite phase. The initial structure is provided for comparison in $(\mathrm{g})$.

\section{Conclusion}

The atomic simulations based on the two-phase model of bcc-Fe and cementite in this work characterize the microscopic mechanism of cumulative damage in bearing steels under RCF. The main conclusions can be summarized as follows.

1. The stress responses of the atomic model appears cyclic softening under all three kinds of cyclic load, and a larger load leads to a greater reduction in stress amplitude and a time-advanced cyclic softening.

2. Dislocations nucleate and annihilate on the interface when shear load amplitude and cycle index are small. When the amplitude and index are large enough, regular structures of the material are destroyed, and massive dislocations retain in the model, resulting in the gradual accumulation of plasticity.

3. Larger shear load amplitude leads to higher average density of dislocation and larger proportion of irregular structure in the model. As loading direction changes, the stress responses and dislocation density of the model is basically unaffected, but the proportion of irregular structures, as well as the initiation and movement of dislocations changes significantly. 
4. Large shear load amplitude causes a mass of defect meshes in the model, further resulting in lots of residual shear strains and stresses in the model. When the cementite phase tends to be damaged under severe shear deformation, the material is usually in a dangerous state, which indicates the origin of failure. Since cementite is brittle and difficult to deform, it can represent a series of brittle inclusions in bearing steels. Therefore, plastic accumulation and defect initiation are more likely to occur on the interface between inclusions and bearing steel matrix under RCF.

\section{Declaration of Competing Interest}

The authors declare that they have no known competing financial interests or personal relationships that could have appeared to influence the work reported in this paper.

\section{Acknowledgments}

This work was financially supported by the National Natural Science Foundation of China (No. 51922084). All the calculations were supported by HPC Platform, Xi'an Jiaotong University.

\section{DATA AVAILABILITY STATEMENT}

The data that support the findings of this study are available from the corresponding author upon reasonable request.

\section{References}

1. Rycerz P, Olver A, Kadiric A. Propagation of surface initiated rolling contact fatigue cracks in bearing steel. Int J Fatigue.2017;97:29-38.

2. Curd ME, Burnett TL, Fellowes JW, Donoghue J, Withers PJ. The heterogenous distribution of white etching matter (WEM) around subsurface cracks in bearing steels. Acta Mater.2019;174:300-309.

3. Lundberg G, Palmgren A. Dynamic capacity of rolling bearings. J applied Mech trans asme. 1947.

4. Sadeghi F, Jalalahmadi B, Slack T, Raje N, Arakere NK. A Review of Rolling Contact Fatigue. $J$ Tribol-Trans ASME. 2009;131(4):041403.

5. Warhadpande A, Sadeghi F, Evans RD. Microstructural Alterations in Bearing Steels under Rolling Contact Fatigue Part 1-Historical Overview. Tribol Trans. 2013;56(3):349-358.

6. Arakere NK. Gigacycle rolling contact fatigue of bearing steels: A review. Int J Fatigue. 2016;93:238-249.

7. Harris TA, Kotzalas MN. Advanced concepts of bearing technology: Rolling bearing analysis, CRC Press; 2006.

8. Shanyavskiy, A. A. Mechanisms and modeling of subsurface fatigue cracking in metals. Eng Fract Mech.. 2013;110:350-363.

9. Xia Z, Kujawski D, Ellyin F. Effect of mean stress and ratcheting strain on fatigue life of steel. Int $J$ Fatigue.2015;18(5):335-341.

10. Pandkar AS, Arakere N, Subhash G. Ratcheting-based microstructure-sensitive modeling of the cyclic hardening response of case-hardened bearing steels subject to Rolling Contact Fatigue.Int $J$ Fatigue. 2015;73:119-131.

11. Arakere NK, Subhash G. Work hardening response of M50-NiL case hardened bearing steel during shakedown in rolling contact fatigue.Mater Sci Technol. 2012;28(1):34-38.

12. Trojahn W, Valentin P. Bearing steel quality and bearing performance. Mater Sci Technol. 2013;28(1):55-57. 
13. Šmeļova V, Schwedt A, Wang L, Holweger W, Mayer J. Electron microscopy investigations of microstructural alterations due to classical Rolling Contact Fatigue (RCF) in martensitic AISI 52100 bearing steel. Int J Fatigue. 2017;98:142-154.

14. Chakraborty J, Bhattacharjee D, Manna I. Development of ultrafine bainite+martensite duplex microstructure in SAE 52100 bearing steel by prior cold deformation. Scripta Mater. 2009;61(6):604-607.

15. Lobodyuk VA, Meshkov YY, Pereloma EV. On Tetragonality of the Martensite Crystal Lattice in Steels. Metall Mater Trans A.2018;50(1):97-103.

16. Decaudin B, Djega-Mariadassou C, Cizeron G. Structural study of M50 steel carbides. J Alloy Compd. 1995;226(1-2):208-212.

17. Bhadeshia H. Steels for bearings. Prog Mater Sci.2012;57(2):268-435.

18. Lian JL, Zheng LJ, Wang FF, Zhang H. Evolution of carbides on surface of carburized M50NiL bearing steel. J Iron Steel Res Int.2018;25(11):1198-1211.

19. Wang KM, Jing HH, Xu LY, Han YD, Zhao LZ Hu WY, et al. Carbide effects on tensile deformation behavior of [001] symmetric tilt grain boundaries in bcc Fe. Modell Simul Mater Sci Eng..2020;28(3):035006.

20. Liang LW, Wang YJ, Chen Y, Wang HY, Dai LH. Dislocation nucleation and evolution at the ferritecementite interface under cyclic loadings.Acta Mater. 2020;186:267-277.

21. Luu HT, Gunkelmann N. Pressure-induced phase transformations in Fe-C: Molecular dynamics approach. Comput Mater Sci.2019;162:295-303.

22. Ghaffarian H, Taheri AK, Ryu S, Kang K. Nanoindentation study of cementite size and temperature effects in nanocomposite pearlite: A molecular dynamics simulation. Curr Appl Phys.2016;16(9):1015-1025.

23. Ghaffarian H, Taheri AK, Kang K, Ryu S. Molecular Dynamics Simulation Study on the Effect of the Loading Direction on the Deformation Mechanism of Pearlite. Multiscale Sci Eng.2019;1(1):47-55.

24. Guziewski M, Coleman SP, Weinberger CR. Atomistic investigation into the mechanical properties of the ferrite-cementite interface: The Bagaryatskii orientation. Acta Mater. 2017;144.

25. Pandkar AS, Arakere N, Subhash G. Microstructure-sensitive accumulation of plastic strain due to ratcheting in bearing steels subject to Rolling Contact Fatigue. Int J Fatigue.2014;63(1):191-202.

26. Briscoe BJ. Contact mechanics. Tribol Int.1985;19(2):109-110.

27. Moghaddam SM, Sadeghi F, Paulson K, Weinzapfel N, Correns M, Bakolas V, et al. Effect of nonmetallic inclusions on butterfly wing initiation, crack formation, and spall geometry in bearing steels.Int $J$ Fatigue. 2015;80:203-215.

28. Plimpton S. Fast Parallel Algorithms for Short-Range Molecular Dynamics. J Comput Phys. 1995;117(1):1-19.

29. Kim J, Kang K, Ryu S. Characterization of the misfit dislocations at the ferrite/cementite interface in pearlitic steel: An atomistic simulation study. Int J Plast. 2016:302-312.

30. Bhadeshia H. Solution to the Bagaryatskii and Isaichev ferrite-cementite orientation relationship problem. Mater Sci Technol. 2018;34(14):1666-1668.

31. Liyanage LSI, Kim SG, Houze J, et al. Structural, elastic, and thermal properties of cementite (Fe3C) calculated using a modified embedded atom method. Phys Rev B. 2014.

32. Nosé S. A molecular dynamics method for simulations in the canonical ensemble. Mol Phys. $1984 ; 52(2): 255-268$.

33. Hoover WG. Canonical dynamics: Equilibrium phase-space distributions. Phys Rev A. 1985;31(3):1695. 
34. Parrinello M, Rahman A. Polymorphic transitions in single crystals: A new molecular dynamics method. J Appl Phys.1981;52(12):7182-7190.

35. Heyes DM. Pressure tensor of partial-charge and point-dipole lattices with bulk and surface geometries. Phys Rev B: Condens Matter. 1994;49(2):755.

36. Beyerlein IJ, Demkowicz MJ, Misra A, Uberuaga BP. Defect-interface interactions. Prog Mater Sci. 2015;74:125-210.

37. Stukowski, Alexander. Visualization and analysis of atomistic simulation data with OVITO-the Open Visualization Tool. Modell Simul Mater Sci Eng. 2010;18(1):2154-2162.

38. Stukowski A, Bulatov VV, Arsenlis A. Automated identification and indexing of dislocations in crystal interfaces. Modell Simul Mater Sci Eng. 2012;20(8):085007.

39. Honeycutt JD, Andersen HC. Molecular dynamics study of melting and freezing of small Lennard-Jones clusters. J Phys Chem.1987;91(19):4950-4963.

40. Faken D, Jónsson H. Systematic analysis of local atomic structure combined with 3D computer graphics. Comput Mater Sci.1994;2(2):279-286.

41. Yuan XY, Yu WW, Fu SC, Yu DJ,Chen X. Effect of mean stress and ratcheting strain on the low cycle fatigue behavior of a wrought 316LN stainless steel. Mater Sci Eng A. 2016; 677:193-202.

42. Kang GZ, Dong YW, Liu YJ, Wang H, Cheng XJ. Uniaxial ratchetting of 20 carbon steel: Macroscopic and microscopic experimental observations. Mater Sci Eng A. 2011;528(16-17):5610-5620.

43. Kuhlmann-Wilsdorf D, Laird C. Dislocation behavior in fatigue.Mater Sci Eng. 1977;27(2):137-156.

44. Guziewski M, Coleman SP, Weinberger CR. Atomistic investigation into interfacial effects on the plastic response and deformation mechanisms of the pearlitic microstructure. Acta Mater. 2019;180:287-300.

45. Fan YH, Wang WY, Hao ZP, Zhan CY. Work hardening mechanism based on molecular dynamics simulation in cutting Ni-Fe-Cr series of Ni-based alloy. J Alloy Compd. 2020;819.

46. Matthews JW, Blakeslee AE. Defects in Epitaxial Multilayers. I. Misfit Dislocations. J Cryst Growth. 1974;27:118-125.

47. Stukowski A, Albe K. Extracting dislocations and non-dislocation crystal defects from atomistic simulation data. Modell Simul Mater Sci Eng. 2010;119(8):2131-2145.

48. Zhang YQ, Jiang SY, Zhu XM, Zhao YN. Mechanisms of crack propagation in nanoscale single crystal, bicrystal and tricrystal nickels based on molecular dynamics simulation. Results Phys. 2017;7:1722-1733.

49. Liang LW, Xiang L, Wang YJ, Chen Y, Wang HY, Dai LH. Ratchetting in cold-drawn pearlitic steel wires. Metall Mater Trans A.2019;50(10):4561-4568.

50. Shibanuma K, Aihara S, Ohtsuka S. Observation and Quantification of Crack Nucleation in FerriteCementite Steel. ISIJ Int . 2013;99(9):582-591. 